\title{
Prevalência da infecção pelo HTLV-1, em remanescentes de quilombos no Brasil Central
}

\author{
Prevalence of infection due to HTLV-1 in remnant quilombos \\ in Central Brazil
}

\author{
Laura Branquinho do Nascimento ${ }^{1}$, Megmar Aparecida dos Santos Carneiro ${ }^{1}$, Sheila Araújo Teles ${ }^{2}$, \\ Carmen Luci Rodrigues Lopes ${ }^{2}$, Nádia Rúbia da Silva Reis ${ }^{1}$, Ágabo Macedo da Costa e Silva ${ }^{1}$, \\ Ana Rita Coimbra Motta-Castro ${ }^{3}$, Koko Otsuki ${ }^{4}$, Ana Carolina Paulo Vicente ${ }^{4}$ \\ e Regina Maria Bringel Martins ${ }^{1}$
}

\begin{abstract}
RESUMO
O presente estudo teve como objetivo estimar a prevalência da infecção pelo HTLV-1 em remanescentes de comunidades de população negra, os quilombos no Brasil Central. 1.837 indivíduos foram avaliados, sendo nove soropositivos para HTLV-1/2 por ELISA. Todos esses foram positivos para HTLV-1 por Western blot e/ou PCR, resultando em uma prevalência de 0,5\% (IC 95\%: 0,2-1,0). A idade dos indivíduos infectados variou de 11 a 82 anos, sendo a maioria do sexo feminino. Quanto às características de risco, história de aleitamento materno, transfusão de sangue, múltiplos parceiros sexuais e doenças sexualmente transmissíveis foram relatadas por esses indivíduos. Os achados deste estudo evidenciam a importância da identificação dos indivíduos infectados pelo HTLV-1 na estratégia de controle e prevenção dessa infecção em remanescentes de quilombos.
\end{abstract}

Palavras-chaves: Vírus linfotrópico de células T humanas 1. Remanescentes de quilombos. Brasil Central. Prevalência.

\section{ABSTRACT}

This study aimed to determine the prevalence of HTLV-1 infection among remnant black quilombo communities in Central Brazil. A total of 1,837 individuals were evaluated, among whom nine were HTLV-1/2 seropositive according to ELISA. All of them were positive for HTLV-1 by means of Western blot and/or PCR, thus resulting in a prevalence of $0.5 \%$ (95\% CI: 0.2-1.0). The HTLV-1 infected individuals ranged in age from 11 to 82 years. The majority of them were females. Regarding risk characteristics, histories of breastfeeding, blood transfusion, multiple sexual partners and sexually transmitted diseases were reported by these individuals. The findings from this study indicate the importance of identifying HTLV-1 infected individuals, as a strategy for infection control and prevention in these remnant quilombos.

Key-words: Human T-cell lymphotropic virus 1. Remnant quilombo. Central Brazil. Prevalence.

A infecção pelo vírus linfotrópico de células T humanas 1 (HTLV-1) está associada com desenvolvimento de doenças graves como a leucemia/linfoma de células T do adulto (ATL) e mielopatia associada ao HTLV-1/paraparesia espástica tropical (HAM/TSP), além de síndromes inflamatórias e complicações infecciosas ${ }^{24}$. Estima-se que 15 a 25 milhões de indivíduos estejam infectados pelo HTLV-1 em todo o mundo. A soroprevalência desse vírus varia de acordo com a área geográfica, grupo étnico e comportamentos de risco. Taxas elevadas de prevalência foram

1. Instituto de Patologia Tropical e Saúde Pública, Universidade Federal de Goiás, Goiânia, GO. 2. Faculdade de Enfermagem, Universidade Federal de Goiás, Goiânia, GO. 3. Departamento de Farmácia Bioquímica, Universidade Federal de Mato Grosso do Sul, Campo Grande, MS. 4. Instituto Oswaldo Cruz, Fundação Oswaldo Cruz, Rio de Janeiro, RJ

Suporte Financeiro: UNESCO/MS-Programa DST-Aids

Endereço para correspondência: Prof $\mathrm{f}$ Regina Maria Bringel Martins. IPTSP/UFG. Caixa Postal 131, 74605-050 Goiânia, G0.

Tel: 5562 3209-6129; Fax: 5562 3209-6363

e-mail: rbringel@terra.com.br

Recebido para publicação em 06/08/2009

Aceito em 27/10/2009 observadas em algumas regiões como o sudoeste do Japão, Melanésia, África equatorial, Caribe e América do Sul ${ }^{20}$.

o Brasil é o país com maior número absoluto de indivíduos infectados por HTLV-1, possuindo cerca de dois milhões de portadores ${ }^{3}$. Essa infecção é endêmica em Salvador, Bahia, onde uma prevalência de 1,8\% foi encontrada ${ }^{8}$. Segundo Alcântara e cols ${ }^{1}$, a introdução do HTLV-1 nesse estado ocorreu com 0 tráfico de escravos africanos. Estudo sobre HTLV-1/2, realizado em doadores de sangue nas capitais dos Estados brasileiros e Distrito Federal, obteve soroprevalência de $0,1 \%$ a $1 \%$, sendo superior nas Regiões Norte e Nordeste, e inferior no sul do Brasil. $\mathrm{Na}$ Região Centro-Oeste, a prevalência verificada nessa população variou de $0,2 \%$ a $0,7 \%$. Em gestantes, uma prevalência de $0,1 \%$ foi observada para o HTLV-1 em Mato Grosso do Sul e Goiás ${ }^{78}$, e de $0,2 \%$ para HTLV- $1 / 2$ em Mato Grosso $0^{25}$.

As comunidades remanescentes de quilombos semi-isoladas no Brasil Central foram fundadas por africanos escravizados, que fugiram para locais de difícil acesso, e instituíram sociedades alternativas conhecidas como quilombos ${ }^{21}$. Considerando a possível origem africana do HTLV-1 e a escassez de estudos 
epidemiológicos para esse vírus nessas comunidades ${ }^{22}$, bem como a inexistência de dados sobre essa infecção em remanescentes de quilombos no Brasil Central, o presente estudo teve como objetivo estimar a prevalência da infecção pelo HTLV-1 nessa população.

\section{MATERIAL E MÉTODOS}

Estudo transversal realizado em remanescentes de quilombos em Goiás e Mato Grosso do Sul, Brasil Central, cuja população totalizava 4.500 indivíduos, no período de julho de 2004 a julho de 2005. Para obter uma prevalência estimada de 0,3\%, com precisão de $0,2 \%$, fez-se necessário estudar o mínimo de 1.757 indivíduos, considerando um poder estatístico de $80 \%(\beta=0,20)$ e nível de significância de $95 \%(\alpha=0,05)$. Assim sendo, a amostra deste estudo foi constituída de 1.837 indivíduos. Este estudo foi aprovado pelo Comitê de Ética em Pesquisa do Hospital Materno Infantil de Goiânia, GO (protocolo CEPHA - HMI n ${ }^{0}$ 025/05).

Todos os indivíduos foram entrevistados sobre dados sóciodemográficos e possíveis fatores associados à infecção pelo HTLV, após assinatura do Termo de Consentimento Livre e Esclarecido, ou da coleta de impressão digital dos indivíduos analfabetos. Para menores de idade, o referido termo foi assinado pelos pais ou responsáveis. Em seguida, amostras sanguíneas (soros) foram coletadas para a realização da triagem sorológica.

A pesquisa de anticorpos anti-HTLV-1/2 foi realizada pelo ensaio imunoenzimático, ELISA (Murex HTLV-I+II, GE80/81, Murex Diagnostics, UK). Após a triagem sorológica, coletou-se uma segunda amostra de sangue dos indivíduos soro reagentes para confirmação por Western blot (WB) (HTLV Blot 2.4, Genelabs Diagnostics, Cingapura) e detecção do DNA proviral. Assim, amostras de sangue total desses indivíduos foram submetidas à extração do DNA utilizando os reagentes da Qiagen (QIAamp DNA Blood Minikit, Qiagen, Hilden, Germany), de acordo com as instruções do fabricante. O DNA proviral foi amplificado por nested-PCR com iniciadores para as regiões tax e long terminal repeat (LTR) do genoma do HTLV-1 ${ }^{1023}$.

Os dados foram analisados pelo programa Epi Info 6 versão 3.04 (2007). A prevalência foi calculada com intervalo de confiança de $95 \%$.

\section{RESULTADOS}

A idade da população estudada variou de 1 a 108 anos, com média (dp) de $29(20,1)$ anos e mediana de 25 anos. A maioria era do sexo feminino $(56,3 \%)$, casada ou com união consensual $(42,1 \%)$, cursou ou estava cursando o ensino fundamental $(62,6 \%)$, lavrador $(45,6 \%)$ e referiu renda menor que um salário mínimo (55\%). Quanto às características de risco para infecção pelo HTLV-1, 28,7\% dos quilombolas relataram mais que cinco parceiros sexuais na vida, 12,9\% referiram antecedentes de doenças sexualmente transmissíveis (DST) e 7,5\% de transfusão de sangue (Tabela 1). Além disso, todos os entrevistados informaram história de aleitamento materno. Por outro lado, não se observou relato de uso de drogas injetáveis nessa população.
TABELA 1

Características sócio-demográficas e de possível risco para infecção pelo HTLV-1, em remanescentes de quilombos estudados no Brasil Central, 2005.

\begin{tabular}{|c|c|c|}
\hline Característica & Número & Percentagem \\
\hline \multicolumn{3}{|c|}{ Média de idade (dp) 29 anos $(20,1)$} \\
\hline \multicolumn{3}{|l|}{ Sexo } \\
\hline feminino & 1.035 & 56,3 \\
\hline masculino & 802 & 43,7 \\
\hline \multicolumn{3}{|l|}{ Estado civil ${ }^{1}$} \\
\hline solteiro & 471 & 34,8 \\
\hline casado/união consensual & 569 & 42,1 \\
\hline separado/viúvo & 312 & 23,1 \\
\hline \multicolumn{3}{|l|}{ Grau de instrução ${ }^{2}$} \\
\hline nenhum & 361 & 22,0 \\
\hline ensino fundamental & 1.026 & 62,6 \\
\hline ensino médio & 222 & 13,6 \\
\hline ensino superior & 29 & 1,8 \\
\hline \multicolumn{3}{|l|}{ Ocupação ${ }^{3}$} \\
\hline lavrador & 578 & 45,6 \\
\hline do lar & 258 & 20,3 \\
\hline estudante & 168 & 13,2 \\
\hline outra & 265 & 20,9 \\
\hline \multicolumn{3}{|c|}{ Renda familiar (salário mínimo) } \\
\hline$<1$ & 1.010 & 55,0 \\
\hline$\geq 1$ & 827 & 45,0 \\
\hline \multicolumn{3}{|l|}{ Parceiros sexuais $\left(\mathrm{n}^{\mathbf{0}}\right)^{4}$} \\
\hline nenhum & 201 & 14,9 \\
\hline$\leq 5$ & 763 & 56,4 \\
\hline$>5$ & 388 & 28,7 \\
\hline \multicolumn{3}{|l|}{ Antecedentes de DST $^{5}$} \\
\hline não & 1.003 & 87,1 \\
\hline sim & 148 & 12,9 \\
\hline \multicolumn{3}{|l|}{ Transfusão de sangue } \\
\hline não & 1.698 & 92,4 \\
\hline sim & 138 & 7,5 \\
\hline sem informação & 1 & 0,1 \\
\hline
\end{tabular}

Dp: desvio padrão. Foram considerados: ${ }^{1}$ indivíduos acima de 12 anos $\left(\mathrm{n}^{0}=1.352\right)$, ${ }^{2}$ indivíduos acima de 6 anos $\left(\mathrm{n}^{\circ}=1.638\right) ;{ }^{3}$ indivíduos acima de 14 anos $\left(\mathrm{n}^{\circ}=1.269\right)$, ${ }^{4}$ indivíduos acima de 12 anos ( $\left.\mathrm{n}^{\circ}=1.352\right),{ }^{5} \mathrm{DST}$ : doenças sexualmente transmissíveis, sendo considerados os indivíduos que relataram atividade sexual $\left(\mathrm{n}^{0}=1.151\right)$.

Das 1.837 amostras testadas, nove foram reagentes para HTLV-1/2 por ELISA e apresentaram reatividade com as proteínas codificadas por gag (p19 e p24) no WB, bem como para as proteínas codificadas pelo envelope (GD21 e rgp46-I), exceto a Q-245 (indeterminada). Verificou-se que as oito amostras positivas no WB foram classificadas como HTLV-1, tendo em vista a reatividade para rgp 46-I, uma proteína recombinante exclusiva do envelope desse vírus. Amostras de sangue total desses indivíduos foram submetidas à detecção do DNA para as regiões tax e LTR do HTLV-1, sendo oito positivas para as duas regiões. A amostra Q-245, com padrão indeterminado no $\mathrm{WB}$, foi positiva para a região tax e negativa para LTR (Tabela 2). Por conseguinte, nove amostras foram positivas nos testes confirmatórios 
TABELA 2

Detecção de anticorpos anti-HTLV (ELISA/Western blot) e do DNA viral pela PCR nas nove amostras soro reagentes.

\begin{tabular}{lcccccc}
\hline & \multicolumn{2}{c}{ Anti-HTLV (ELISA) } & & Anti-HTLV-1 & \multicolumn{2}{c}{ DNA HTL-1 } \\
\cline { 2 - 5 } Amostra & D0 & Cutoff & & (Western blot $)$ & Tax & LTR \\
\hline Q-1 & $>1.0$ & 0.471 & p19,p24, GD21, rgp46-I & + & + \\
Q-71 & $>1.0$ & 0.377 & p19,p24, GD21, rgp46-I & + & + \\
Q-130 & $>1.0$ & 0.409 & p19,p24, GD21, rgp46-I & + & + \\
Q-131 & $>1.0$ & 0.409 & p19,p24, GD21, rgp46-I & + & + \\
Q-132 & $>1.0$ & 0.409 & p19,p24, GD21, rgp46-I & + & + \\
Q-245 & 0.626 & 0.348 & & p19,p24 & + & - \\
Q-344 & $>1.0$ & 0.372 & p19,p24, GD21, rgp46-I & + & + \\
Q-535 & $>1.0$ & 0.369 & p19,p24, GD21, rgp46-I & + & + \\
Q-710 & $>1.0$ & 0.376 & p19,p24, GD21, rgp46-I & + & + \\
\hline
\end{tabular}

Anti-HTLV: anticorpos contra o vírus linfotrópico de células T humanas, D0: densidade ótica. *as diferenças nos valores do cutoff (ponto de corte) foram estabelecidas com os controles positivos e negativos em cada placa do Kit, LTR: long terminal repeat.
(WB e/ou PCR), resultando numa prevalência global de $0,5 \%$ (IC 95\% 0,2-1,0) para infecção pelo HTLV-1.

As características dos indivíduos infectados, inclusive aquelas associadas a risco aumentado da infecção pelo HTLV1, são apresentadas na Tabela 3. A idade variou de 11 a 82 anos, sendo o sexo feminino predominante. Destes, quatro residiam em Goiás e cinco em Mato Grosso do Sul. A maioria dos indivíduos relatou que foi amamentada por mais de seis meses. Dois quilombolas positivos referiram transfusão de sangue, sendo um episódio anterior a 1993 e o outro em 1998. A idade da primeira relação sexual foi informada por seis indivíduos, variando de 13 a 20 anos. 0 número de parceiros sexuais relatado variou de um a mais de 15 . Dois indivíduos informaram múltiplos parceiros, sendo que um mencionou história de DST e não usava preservativo, dentre os infectados pelo HTLV-1.

TABELA 3

Características dos remanescentes de quilombos infectados pelo HTLV-1 no Brasil Central, 2005.

\begin{tabular}{|c|c|c|c|c|c|c|c|c|c|}
\hline \multirow[b]{2}{*}{ Características } & \multicolumn{9}{|c|}{ Remanescentes de quilombos } \\
\hline & Q-1 & Q-71 & Q-130 & Q-131 & Q-132 & Q-245 & Q-344 & Q-535 & Q-710 \\
\hline Idade (anos) & 32 & 11 & 63 & 55 & 27 & 42 & 61 & 82 & 71 \\
\hline Sexo & $\mathrm{F}$ & $\mathrm{F}$ & M & $\mathrm{F}$ & $\mathrm{F}$ & M & $\mathrm{F}$ & $\mathrm{F}$ & $\mathrm{F}$ \\
\hline Localidade & MS & MS & MS & MS & MS & G0 & G0 & G0 & GO \\
\hline Amamentação (meses) & $>6$ & $>6$ & $>6$ & SI & $>6$ & SI & $<6$ & SI & $>6$ \\
\hline Transfusão de sangue & $\mathrm{N}$ & $\mathrm{N}$ & S & $\mathrm{N}$ & $\mathrm{N}$ & $\mathrm{N}$ & $\mathrm{N}$ & S & $\mathrm{N}$ \\
\hline Idade $1^{a}$ relação sexual (anos) & 19 & $\mathrm{NA}$ & 13 & 17 & 16 & SI & 20 & 15 & SI \\
\hline Parceiros sexuais $\left(n^{\circ}\right)$ & 1 & $\mathrm{NA}$ & $>15$ & 1 & 5 & $>15$ & 2 & 2 & 2 \\
\hline História de DST & $\mathrm{N}$ & $\mathrm{N}$ & S & $\mathrm{N}$ & $\mathrm{N}$ & $\mathrm{N}$ & $\mathrm{N}$ & $\mathrm{N}$ & $\mathrm{N}$ \\
\hline
\end{tabular}

F: feminino, M: masculino, MS: Mato Grosso do Sul, GO: Goiás, SI: sem informação, NA: não se aplica, N: não, S: sim.

\section{DISCUSSÃO}

Esta é a primeira investigação epidemiológica do HTLV-1 em remanescentes de quilombos no Brasil Central, cuja população foi composta em sua maioria por indivíduos do sexo feminino, e com níveis baixos de renda familiar e escolaridade. Características semelhantes foram observadas na população em geral de Salvador, constituída predominantemente por afrodescendentes, e se mostraram associadas à infecção pelo HTLV-1 ${ }^{8}$. Provavelmente, a baixa frequiência de quilombolas infectados da presente investigação não permitiu evidenciar a ocorrência dessas associações (dados não mostrados).

A prevalência global da infecção pelo HTLV-1 neste estudo foi concordante com as observadas em doadores de sangue na Região Centro-Oeste ${ }^{4}$ e em remanescentes de quilombos no Pará (1,1\%; IC 95\%: $0,3-3,6)^{22}$. Por outro lado, foi inferior à encontrada na população em geral de Salvador, Bahia (1,8\%; IC 95\%: 1,1-2,5) mas superior às verificadas em gestantes em Goiás (0,1\%; IC 95\%: 0,1-0,2) e Mato Grosso do Sul (0,1\%; IC 95\%: 0,1-0,2) $)^{718}$. De acordo com esses dados, a prevalência para infecção pelo HTLV-1 em remanescentes de quilombos no Brasil Central pode ser considerada intermediária.

No presente estudo, o WB confirmou positividade para o HTLV-1 em oito $(88,9 \%)$ das nove amostras soro reagentes pelo ELISA. Apenas uma amostra foi indeterminada, sendo que esta apresentou reatividade para proteínas codificadas por gag (p19 e p24) e foi PCR positiva para a região tax, mas negativa para LTR. Esse perfil de reatividade pode ocorrer durante 0 período de soroconversão, já que os anticorpos contra gag surgem primeiramente, ou quando da infecção com provírus defectivo do HTLV, ou ainda devido à reatividade cruzada com outros agentes. Dessa forma, faz-se necessária a detecção do DNA viral nas amostras indeterminadas pelo WB, para diagnóstico e acompanhamento dos indivíduos infectados ${ }^{5611}$.

Dentre os remanescentes de quilombos infectados pelo HTLV-1, observou-se maior soropositividade no sexo feminino, sendo concordante com outros estudos ${ }^{81315}$. A frequência elevada de mulheres infectadas pelo HTLV-1 pode ser atribuída à maior eficiência da transmissão sexual do vírus no sentido homemmulher ${ }^{12}{ }^{17}$, podendo ainda favorecer a transmissão vertical por amamentação ${ }^{2919}$. De fato, o aleitamento materno por mais de seis meses foi referido pela maioria dos indivíduos infectados pelo HTLV-1, neste estudo.

Ainda em relação à transmissão sexual desse vírus, fatores como múltiplos parceiros sexuais durante a vida, sexo sem proteção, iniciação sexual precoce e história de DST foram associados à soropositividade ao HTLV-1 15 16 17. Embora tais associações não foram evidenciadas na presente investigação, dois dos nove indivíduos infectados pelo HTLV-1 relataram múltiplos 
parceiros sexuais, sendo que um referiu também antecedentes de DST e sua primeira relação sexual ocorreu aos 13 anos de idade (Q-130).

No Brasil, a hemotransfusão foi um fator de risco significante para a transmissão do HTLV-1, principalmente se ocorreu antes de novembro de 1993, quando foi implantada a triagem sorológica para HTLV-1/2 nos bancos de sangue ${ }^{14}$. Dentre os indivíduos infectados pelo HTLV-1, neste estudo, história de hemotransfusão foi informada por dois quilombolas. Um relatou episódio transfusional anterior ao ano de 1993 (Q-535) e não informou outras características de risco, ratificando a importância da triagem dos doadores de sangue. 0 outro individuo (Q-130), apesar de ter sido transfundido em 1998 (após a implantação da triagem sorológica para esse vírus), o mesmo referiu características de risco relacionadas a transmissão sexual do HTLV-1.

Como os remanescentes de quilombos infectados pelo HTLV-1 vivem em comunidades ainda semi-isoladas, acredita-se que a introdução do vírus possa estar vinculada ao tráfico de escravos da África para o Brasil e, provavelmente, a manutenção da infecção vem ocorrendo pela transmissão vertical e/ou horizontal. Entretanto, investigações moleculares são necessárias para elucidação da possível origem africana do HTLV-1 nas comunidades remanescentes de quilombos no Brasil.

Diante dos achados deste estudo e considerando o risco dos indivíduos infectados desenvolverem doenças graves como ATL e HAM/TSP, além de outras associadas ${ }^{24}$, medidas incluindo a triagem para HTLV durante o pré-natal, aconselhamento das gestantes infectadas em relação à suspensão da amamentação, ou a redução da mesma pelo fornecimento de fórmulas infantis, devem ser implementadas. Adicionalmente, faz-se necessária a adoção de estratégias para 0 incentivo à prática sexual segura, como o uso regular de preservativo.

\section{REFERÊNCIAS}

1. Alcantara LCJ, Oliveira T, Gordon M, Pybus O, Mascarenhas RE, Seixas MO, Gonçalves M, Hlela C, Cassol S, Galvão-Castro B. Tracing the origin of Brazilian HTLV-1 as determined by analysis of host and viral genes. AIDS 20: 780-782, 2006.

2. Ando Y, Ekuni Y, Matsumoto Y, Nakano S, Saito K, Kakimoto K, Tanigawa T, Kawa M, Toyama T. Long-term serological outcome of infants who received frozen-thawed milk from human T-lymphotropic virus type-I positive mothers. Journal of Obstetrics and Gynaecollogy Research 30: 436-438, 2004.

3. Carneiro-Proietti AB, Catalan-Soares B, Proietti FA. Human T cell lymphotropic viruses (HTLV-I/II) in South America: should it be a public health concern? Journal of Biomedical Science 9: 587-595, 2002.

4. Catalan-Soares B, Carneiro-Proietti AB, Proietti FA. Heterogeneous geographic distribution of human T-cell lymphotropic viruses I and II (HTLV-I/II): serological screening prevalence rates in blood donors from large urban areas in Brazil. Caderno de Saúde Pública 21: 926-931, 2005.

5. Césaire R, Bera 0, Maier H, Lezin A, Martial J, Ouka M, Kerob-Bauchet B, Ould Amar AK, Vernant JC. Seroindeterminate patterns and seroconversions to human T-lymphotropic virus type I positivity in blood donors from Martinique, French West Indies. Transfusion 39: 1145-1149, 1999.

6. Costa JMP, Segurado AC. Molecular evidence of human T-cell lymphotropic virus types 1 and 2 (HTLV-1 and HTLV-2) infections in HTLV seroindeterminate individuals from São Paulo, Brazil. Journal of Clinical Virology 44: 185-189, 2009.

7. Dal Fabbro MMFJ, Cunha RV, Boia MN, Portela P, Botelho CA, Freitas GMB, Soares J, Ferri J, Lupion J. Infecção pelo HTLV 1/2: atuação no pré-natal como estratégia de controle da doença no Estado de Mato Grosso do Sul. Revista da Sociedade Brasileira de Medicina Tropical 41: 148-151, 2008.

8. Dourado I, Alcantara LCJ, Barreto ML, Teixeira MG, Galvao-Castro B. HTLV-I in the general population of Salvador, Brazil: a city with African ethnic and sociodemographic characteristics. Journal Acquired Immune Deficiency Syndromes 34: 527-531, 2003.

9. Fujino T, Nagata Y. HTLV-I transmission from mother to child. Journal Reproductive Immunology 47: 197-206, 2000.

10. Furukawa Y, Yamashita M, Usuku K, Izumo S, Nakagawa M, Osame M. Phylogenetic subgroups of human T cell lymphotropic virus (HTLV) type I in the tax gene and their association with different risks for HTLV-I-associated myelopathy/tropical spastic paraparesis. The Journal of Infectious Diseases 182:1343-1349, 2000.

11. Gessain A, Mathieux R. HTLV-I "indeterminate” western blot patterns observed in sera from tropical regions: the situation revisited. Journal of Acquired Immune Deficiency Syndromes and Human Retrovirology 9: 316-319, 1995.

12. Kaplan JE, Khabbaz RF, Murphy EL, Hermansen S, Roberts C, Lal R, Heneine W, Wright D, Matijas L, Thomson R, Rudolph D, Switzer WM, Kleinman S, Busch M, Schreiber GB. Male-to-female transmission of human T-cell lymphotropic virus types I and II: association with viral load. The Retrovirus Epidemiology Donor Study Group. Journal of Acquired Immune Deficiency Syndromes and Human Retrovirology 12: 193-201, 1996.

13. Larsen 0, Andersson S, Silva Z, Hedegaard K, Sandstrom A, Naucler A, Dias F, Melbye M, Aaby P. Prevalences of HTLV-1 infection and associated risk determinants in an urban population in Guinea-Bissau West Africa. Journal of Acquired Immune Deficiency Syndromes 25: 157-163, 2000.

14. Ministério da Saúde. Portaria $n^{0} 1.376$ em 19 de novembro de 1993. Disponível em: http://www.anvisa.gov.br/legis/portarias/1376-93.pdf. Acesso em 15 de novembro de 2008.

15. Mota A, Nunes C, Melo A, Romeo M, Boasorte N, Dourado I, Alcântara LC, GalvãoCastro B. A case-control study of HTLV-infection among blood donors in Salvador, Bahia, Brazil - Associated risk factors and trend towards declining prevalence. Revista Brasileira de Hematologia e Hemoterapia 28: 120-126, 2006.

16. Moxoto I, Boa-Sorte N, Nunes C, Mota A, Dumas A, Dourado I, Galvão-Castro B. Perfil sócio-demografico, epidemiológico e comportamental de mulheres infectadas pelo HTLV-1 em Salvador, Bahia, uma área endêmica para o HTLV. Revista da Sociedade Brasileira de Medicina Tropical 40: 37-41, 2007.

17. Murphy EL, Figueroa JP, Gibbs WN, Brathwaite A, Holding-Cobham M, Waters D, Cranston B, Hanchard B, Blattner WA. Sexual transmission of human T-lymphotropic virus type I (HTLV-I). Annals of Internal Medicine 111: 555-560, 1989.

18. Oliveira SR, Avelino MM. Soroprevalência do vírus linfotrópico - T humano tipo I entre gestantes em Goiânia, GO, Brasil. Revista Brasileira de Ginecologia e Obstetrícia 28: 467-472, 2006.

19. Pimenta FC, Kashima Haddad S, de Medeiros Filho JG, Costa MJC, Diniz MFM, Fernandes MP, de Araujo LB, Pombo-de-Oliveira MS. Prevalence ratio of HTLV-1 in nursing mothers from the state of Paraiba, Northeastern Brazil. Journal of Human Lactation 24: 289-292, 2008

20. Proietti FA, Carneiro-Proietti AB, Catalan-Soares BC, Murphy EL. Global epidemiology of HTLV-I infection and associated diseases. Oncogene 24: 6058-6068, 2005.

21. Secretaria Especial de Políticas de Promoção da Igualdade Racial (Seppir) 2008. Disponível em: http://www.presidencia.gov.br/estrutura_presidencia/seppir/ noticias/ultimas_noticias/. Acesso em 09 de outubro de 2008

22. Vallinoto ACR, Pontes GS, Muto NA, Lopes IGL, Machado LFA, Azevedo VN, Carvalhaes FAPL, Santos SEB, Guerreiro JF, Ishak MOG, Ishak R . Identification of human T-cell lymphotropic virus infection in a semi-isolated Afro-Brazilian quilombo located in the Marajo Island (Para, Brazil). Memórias do Instituto Oswaldo Cruz 101: 103-105, 2006.

23. Vandamme AM, Liu HF, Goubau P, Desmyter J. Primate T-lymphotropic virus type I LTR sequence variation and its phylogenetic analysis: compatibility with an African origin of PTLV-1. Virology 202: 212-223, 1994.

24. Verdonck K, Gonzalez E, Van Dooren S, Vandamme AM, Vanham G, Gotuzzo E. Human T-lymphotropic virus 1: recent knowledge about an ancient infection. The Lancet Infectious Disease 7: 266-281, 2007.

25. Ydy RRA, Ferreira D, Souto FJD, Fontes CJF. Prevalência da infecção pelo vírus linfotrópico humano de células T-HTLV1/2 entre puérperas de Cuiabá, Estado de Mato Grosso, 2006. Revista da Sociedade Brasileira de Medicina Tropical 42: 28-32, 2009. 